\title{
Resección intestinal por endometriosis severa, diagnóstico y tratamiento luego de la introducción de la Unidad de Endometriosis: revisión de 18 años en el Hospital Clínico de la Universidad de Chile
}

\section{Bowel resection for severe endometriosis, diagnosis and treatment after the introduction of Endometriosis Unit: 18 years review, Clinical Hospital of the University of Chile}

\author{
Ignacio Miranda-Mendoza1,2*, Paz Navarrete-Rey ${ }^{1,3}$, Natalia Moreno $^{4}$, Luis Aravena², Mauricio Díaz y \\ Mario Abedrapo 4
}

${ }^{1}$ Departamento de Obstetricia y Ginecología, Facultad de Medicina, Universidad Del Desarrollo, Clínica Alemana de Santiago, Santiago; ${ }^{2}$ Unidad de Endometriosis, Departamento de Obstetricia y Ginecología, Hospital Clínico, Universidad de Chile, Santiago; ${ }^{3}$ Unidad de Ginecología, Complejo Asistencial Padre Las Casas, Temuco; ${ }^{4}$ Unidad de Endometriosis, Coloproctología, Departamento de Cirugía, Hospital Clínico, Universidad de Chile, Santiago. Chile

\section{Resumen}

Introducción: La endometriosis intestinal afecta en gran medida la calidad de vida de una mujer joven y habitualmente requiere un tratamiento quirúrgico con resección intestinal. Esta cirugía es técnicamente compleja por las adherencias firmes del intestino a la vagina, el útero y los ovarios. Objetivo: Describir y analizar los resultados quirúrgicos e histopatológicos de las resecciones intestinales por endometriosis grave durante los últimos 18 años en el Hospital Clínico de la Universidad de Chile, en relación con la introducción de la unidad multidisciplinaria de endometriosis, a partir del año 2011, y las experiencias publicadas en la literatura chilena y extranjera. Método: Trabajo retrospectivo realizado en un hospital terciario desde el año 2001 hasta el año 2019. Las pacientes se asignaron a dos grupos según el período de cirugía: grupo 2001-2010 y grupo 2011-2019, luego de la introducción de la unidad de endometriosis. Se recopilaron todas las pacientes a las que se realizó una resección intestinal (discoidal o segmentaria) por endometriosis, por laparotomía o laparoscopía. Los datos distribuidos normalmente se presentan como promedio $\pm D E$ y los datos no paramétricos como mediana (rango). Las comparaciones demográficas de variables continuas se hicieron con la prueba t de Student y las de las variables categóricas con las pruebas de ji al cuadrado o de Fisher. La significación estadística se estableció en $p<0,05$. Resultados: Se recopilaron 52 casos. El 94,2\% de las cirugías fueron electivas. El 5,8\% fueron de urgencia por obstrucción intestinal (todas entre 2001 y 2010). Un 75\% de las cirugías fueron laparoscópicas. Se realizó resección segmentaria en el 67,3\%, resección discoidal simple en el 28,8\%, resección discoidal doble en el 1,9\% y resección segmentaria y una discoidal en el 1,9\%. La histopatología demostró compromiso de la lesión hasta la mucosa intestinal en un 7,7\%. Hubo franca disminución del dolor en el seguimiento de las pacientes. El $24 \%$ de las pacientes con deseo de embarazo y endometriosis intestinal lograron un parto de término mediante fecundación in vitro o espontáneamente. Hubo cuatro complicaciones posoperatorias, tres de ellas de categoría II según la clasificación de Clavien-Dindo y una de categoría IV A con reintervención a las 72 horas. Al comparar ambos periodos, en 2001-2010 los exámenes diagnósticos utilizados fueron ecografía transvaginal (0\%), enema baritado (60\%), tomografía computarizada de abdomen y pelvis (45\%) y resonancia magnética pelviana (20\%), mientras que en 2011-2019 fueron ecografía transvaginal (100\%), enema baritado (3\%), tomografía computarizada (3\%) y resonancia magnética pelviana (66\%). En 2001-2010, las lesiones fueron más más infiltrativas (mayor compromiso mucoso y submucoso)

orrespondencia:

*Ignacio Miranda-Mendoza

E-mail: imiranda@hcuch.cl
Disponible en internet: 02-08-2021 Rev Chil Obstet Ginecol. 2021;86(3):291-300

www.rechog.com 0048-766X / @ 2021 Sociedad Chilena de Obstetricia y Ginecología. Publicado por Permanyer. Éste es un artículo open access bajo la licencia CC BY-NC-ND (https://creativecommons.org/licenses/by-nc-nd/4.0/). 
Rev Chil Obstet Ginecol. 2021;86(3)

( 75 vs. $16 \%$ de las resecciones intestinales; $p<0,05$ ), estenóticas (cirugías de urgencia por obstrucción), con mayor porcentaje de resecciones segmentarias (100 vs. 46,9\%; $p$ < 0,05) y más días de hospitalización $(5,8 \pm 2,3$ vs. 4,1 \pm 0,9 días) que en 2011-2019. Conclusiones: A nuestro entender, esta es la serie más grande publicada en Chile de resecciones intestinales por endometriosis. Estos hallazgos demuestran cómo la introducción de la unidad multidisciplinaria de endometriosis permite un diagnóstico precoz y un tratamiento quirúrgico eficaz y oportuno, tal como se decribe en la literatura.

Palabras clave: Endometriosis. Dismenorrea. Cirugía laparoscópica. Enfermedad ginecológica. Endometriosis intestinal.

\section{Abstract}

Introduction: Bowel endometriosis severely affects a young woman's quality of life and often requires surgical treatment with bowel resection. This surgery is technically complex due to the tight adhesions of the intestine to the vagina, uterus, and ovaries. The objective of this work is to describe and analyze the surgical and histopathological results of intestinal resections for severe endometriosis during the last 18 years at the Clinical Hospital University of Chile, in relation to the implementation of the multidisciplinary endometriosis unit, based on the year 2011 and the experiences published in Chilean and foreign literature. Method: Retrospective work carried out in a tertiary hospital from 2001 to 2019. The patients were assigned to two groups according to the surgery period: group 2001-2010 and group 2011-2019, after endometriosis unit formation. All patients who underwent bowel resection (discoidal or segmental) for endometriosis by laparotomy or laparoscopy were collected. Normally distributed data are presented as mean $\pm S D$ and nonparametric data as median (range). Demographic comparisons of continuous variables are compared using Student's $t$ test and categorical variables using chi squared or Fisher's test. Statistical significance was established at $p<0.05$. Results: 52 cases were collected. $94.2 \%$ of the surgeries were elective. 5.8\% were urgent due to intestinal obstruction (all between 2001 and 2010). $75 \%$ of the surgeries were laparoscopic. Segmental resection $67.3 \%$, simple discoidal resection $28.8 \%$, double discoidal resection $1.9 \%$ and segmental resection and a discoidal resection $1.9 \%$. Histopathology showed involvement of the lesion up to the intestinal mucosa in $7.7 \%$. A marked decrease in pain in the follow-up of the patients. $24 \%$ of the patients with a desire for pregnancy and intestinal endometriosis achieved a full-term delivery by IVF or spontaneously. There were four postoperative complications, three of them category II according to the Clavien-Dindo classification, and one category IV A complication with reoperation at $72 \mathrm{~h}$. When comparing both periods, between 2001-2010 the diagnostic tests used were: transvaginal ultrasound (ECO TV) (0\%), barium enema (BE) (60\%), abdomen pelvis CT (45\%) and pelvic resonance (MRI) (20\%). Between 2011 and 2019 ECO TV (100\%), EB (3\%), TAC (3\%) RM (66\%). In the period 2001 to 2010, the lesions were more infiltrative (greater mucosal and submucosal involvement) $(75 \%$ vs $16 \%$ of intestinal resections $(P<0.05))$, stenotic (urgent surgery for obstruction), with a higher percentage of resections segmental $(100 \%$ vs $46.9 \%(P<0.05)$ and more days of hospitalization $(5.8 \pm 2.3 S D$ vs $4.1 \pm 0.9 S D)$ than in the period from 2011 to 2019. Conclusions: To our knowledge, this is the largest series published in Chile of intestinal resections for endometriosis. These findings demonstrate how the introduction of the multidisciplinary endometriosis unit allows early diagnosis and effective and timely surgical treatment as described in the literature.

Key words: Endometriosis. Dysmenorrhea. Laparoscopic surgery. Gynecologic disease. Bowel endometriosis.

\section{Introducción}

La endometriosis es una enfermedad que se caracteriza por la presencia de tejido endometrial fuera de la cavidad uterina y afecta alrededor del $10-15 \%$ de las mujeres en edad reproductiva ${ }^{1-4}$. Se ubica fundamentalmente en la pelvis, los ovarios y el peritoneo superficial y profundo. La endometriosis profunda es aquella que infiltra el peritoneo en más de $5 \mathrm{~mm}$, con una lesión característica que es el nódulo endometriósico retroperitoneal, el cual puede estar ubicado en la vagina, la vejiga, el intestino, el uréter 0 , en ocasiones, ser multifocal ${ }^{1,2,4}$. Su cuadro clínico habitual son síntomas de dolor pélvico, como dismenorrea (dolor durante la menstruación), dolor pelviano no cíclico, dispareunia (dolor en la actividad sexual), disquecia (dolor en la defecación), rectorragia catamenial e infertilidad. En ocasiones puede presentarse con complicaciones más graves, como obstrucción intestinal, hidronefrosis y disfunción renal ${ }^{5-8}$.

La endometriosis intestinal afecta al $15-20 \%$ de las pacientes con endometriosis profunda, infiltrando principalmente el recto y la unión rectosigmoidea en un $60-70 \%$, el colon sigmoides en un $15-20 \%$, y el ciego, el apéndice y la válvula ileocecal en un 5-10\%6,9,10.

En la endometriosis en general y en aquellas con compromiso intestinal, la primera línea de tratamiento es el médico, el cual incluye el uso de hormonas, analgésicos y antiinflamatorios. Los más utilizados son los 
tratamientos hormonales, como los anticonceptivos orales combinados y las progestinas. Estos tienen una buena tolerancia y bajo costo, y han demostrado lograr una disminución del dolor y eventualmente una ralentización de la progresión de la enfermedad ${ }^{4}$. Sin embargo, en algunos casos se requiere un tratamiento quirúrgico resectivo, principalmente en pacientes con dolor pélvico intenso (puntaje de dolor en la escala visual análoga $[E V A]>7$ de 10), pacientes con diagnóstico de infertilidad con dos o más ciclos frustros de fertilización in vitro (FIV), pacientes que no responden al tratamiento médico y pacientes con signos de obstrucción intestinal independientemente de los síntomas ${ }^{2,5-7}$.

La cirugía en la endometriosis profunda es compleja, debido a la gran distorsión anatómica, la presencia de tejido fibrótico y la obliteración de los fondos de sacos rectovaginal y vesicouterino ${ }^{1,2,5,11}$. Esto hace que la cirugía sea laboriosa, con pasos establecidos para la restauración anatómica (liberación de adherencia fisiológica del sigmoides, liberación de los anexos, ureterólisis unilateral o bilateral, apertura de ambas fosas pararrectales y resección del nódulo retroperitoneal) y asociada a tasas de complicaciones del $3,5 \%$ al $25 \%$ $2,8,11-15$.

Los mejores resultados se obtienen equilibrando la radicalidad de la resección de la enfermedad con una adecuada preservación de la funcionalidad de los órganos pélvicos de una mujer joven. Para ello se requiere un ginecólogo experto en endometriosis apoyado con equipos profesionales multidisciplinarios especialistas en esta enfermedad, que incluyan urólogos, coloproctólogos, cinesiólogos y médicos de la unidad del dolor ${ }^{1,2,12,16-18}$.

Los reportes nacionales son pocos y muestran la experiencia de algunas series de casos de endometriosis intestinal ${ }^{16,19-21}$. A nuestro entender, las dos más grandes, con 17 y 22 resecciones intestinales, son las de Mordojovich et al. ${ }^{18}$, y Larraín et al. ${ }^{19}$, respectivamente.

En este contexto, el objetivo general de nuestro trabajo es describir y analizar los resultados quirúrgicos e histopatológicos de las resecciones intestinales por endometriosis grave durante los últimos 18 años en un centro terciario universitario, en relación con la introducción de la unidad multidisciplinaria de endometriosis a partir del año 2011 y las experiencias publicadas en la literatura chilena y extranjera. Como objetivos secundarios se evalúan el estudio diagnóstico, la localización de la lesión endometriósica intestinal, el tipo de resección intestinal, las reintervenciones y la infiltración del nódulo endometriósico en la pared intestinal.

\section{Método}

Estudio descriptivo retrospectivo de las resecciones intestinales por endometriosis profunda operadas por vía laparotómica y laparoscópica entre 2001 y 2019 en el Hospital Clínico de la Universidad de Chile. El estudio fue aprobado por el comité de ética científico de investigación del Hospital Clínico de la Universidad de Chile el 18 de diciembre de 2019 (N. ${ }^{\circ}$ 59/19).

Mediante un proceso de anonimización de datos, se recolectaron los casos de todas las pacientes operadas de endometriosis con resección intestinal. Se realizó una búsqueda considerando el diagnóstico operatorio de endometriosis, endometriosis intestinal, endometriosis profunda 0 endometriosis grave en el registro clínico del pabellón de maternidad y del pabellón central. Además, se comparó la información con los códigos quirúrgicos de resección intestinal.

Una vez obtenidos los casos, se recopilaron los protocolos quirúrgicos, los informes de biopsia y los antecedentes clínicos de los registros en ficha clínica de papel desde el año 2001 hasta el año 2010, y del registro clínico electrónico desde 2011 hasta 2019. Se realizó una base de datos en Excel para su análisis. Se incluyeron todos los casos en los que se realizó una resección intestinal por endometriosis confirmada por histología. Se excluyeron aquellas pacientes en que, teniendo diagnóstico de endometriosis, no se realizó resección intestinal. Se hizo seguimiento telefónico en algunos casos, después de 2011, por faltar algunos datos.

Se realizó el test de Shapiro-Wilk para determinar la distribución de variables continuas. Los datos con distribución normal se presentan como promedio \pm DE y los datos no paramétricos como mediana (rango). Las pacientes se asignaron a dos grupos según el periodo de cirugía: grupo 2001-2010 y grupo 2011-2019, luego de la introducción de la unidad de endometriosis. Las variables categóricas se informan como valores absolutos y porcentajes. Las comparaciones demográficas de variables continuas se hicieron con la prueba $t$ de Student y las variables categóricas con las pruebas de ji al cuadrado o de Fisher. La significación estadística se estableció en $p<0,05$. Todos los análisis estadísticos se realizaron con el programa STATA $₫ 16.1$ (Statacorp, College Station, Texas, USA). 


\section{Resultados}

Se encontraron 52 pacientes operadas de endometriosis profunda a las que se realizó una resección intestinal. Se obtuvieron el $100 \%$ de los ingresos preoperatorios, protocolos quirúrgicos e informes anatomopatológicos, pero solo se pudieron recopilar los controles posoperatorios realizados desde 2011, que fue cuando comenzó el registro clínico electrónico.

\section{Características de las pacientes, síntomas y estudio preoperatorio}

Las características epidemiológicas de las pacientes se describen en la tabla 1. El promedio de edad fue de 34,5 años $\pm 5,4$. El 90,4\% presentaba dolor pélvico grave al momento de la cirugía. Las medianas de los síntomas de dolor pelviano en relación a la EVA de dolor fueron: dolor pélvico 7 (rango: 0-10), dismenorrea 9 (rango: 5-10), disquecia 6 (rango: 0-10) y dispareunia 6 (rango: $0-10$ ). El $32,7 \%$ de los casos asociaban infertilidad (Tabla 1). El 34,6\% de las pacientes refirieron rectorragia. El 34,6\% tenían algún tipo de cirugía previa por endometriosis (apendicectomía, quistectomía ovárica, resección de focos).

Respecto al estudio preoperatorio, es importante mencionar que entre 2001 y 2010 el estudio se realizaba principalmente mediante tomografía computarizada (TC) de abdomen y pelvis (45\%), junto con un enema baritado $(60 \%)$, dado que la resonancia magnética (RM) (20\%) era costosa y poco accesible. En el periodo 2011-2019 se comienza a utilizar con frecuencia la RM $(66 \%)$ debido a la disminución de su costo y la mayor accesibilidad. Además, estas se evalúan y se discuten en conjunto con los radiólogos enfocados en esta patología. Desde el año 2011 todas las pacientes tienen ecografía transvaginal y a partir del año 2015 se implementa la ecografía transvaginal extendida para endometriosis profunda, gracias a la formación específica de ginecólogos en esta área y los avances de la tecnología de ultrasonido. Los exámenes de enema baritado y TC bajan en su porcentaje de utilización a un $3 \%$ y un $6 \%$, respectivamente, en este último periodo (Tabla 2).

En relación a su seguimiento, tal como ya se dijo, solo se obtuvieron los controles posoperatorios a partir del año 2011. Estas 32 pacientes tuvieron entre uno y cinco controles registrados en la ficha clínica electrónica; solo un caso no fue registrado. El primer control tras la cirugía fue entre 7 y 14 días posteriores al alta, como es habitual en el servicio. La mediana de
Tabla 1. Características de las pacientes y sus síntomas

\begin{tabular}{|l|c|}
\hline Características pacientes & $\mathrm{n}=52$ \\
\hline Edad promedio, años & $34,5 \pm 5,4$ \\
\hline Infertilidad & $17(32,7)$ \\
\hline Dolor pélvico crónico & $47(90,4)$ \\
\hline Rectorragia & $18(34,6)$ \\
\hline Cirugía de urgencia por obstrucción & $3(5,8)$ \\
\hline Colonoscopía previa & $23(44,2)$ \\
\hline Tratamiento hormonal previo & $20(38,4)$ \\
\hline Puntaje dolor pélvico crónico en EVA & $7 / 10(0-10)$ \\
\hline Puntaje dismenorrea en EVA & $9 / 10(5-10)$ \\
\hline Puntaje disquecia en EVA & $6 / 10(0-10)$ \\
\hline Puntaje dispareunia en EVA & $6 / 10(0-10)$ \\
\hline
\end{tabular}

EVA: escala visual análoga.

Las variables categóricas se expresan como $n(\%)$, las variables continuas se expresan como promedio \pm desviación estándar, y las variables no paramétricas como medianas.

seguimiento fue de 3 meses (rango: 0-15 años). En la primera evaluación posoperatoria se observa en la muestra $(n=32)$ una franca mejoría del dolor pelviano (mediana de puntaje EVA: 2/10). Con el transcurso del tiempo, solo dos casos refirieron persistir con dolor a los 3 años desde la realización de la cirugía. De las pacientes con deseo de fertilidad (17/32), dos lograron un embarazo espontáneo de término, ambas operadas mediante cesárea debido a una distocia de la presentación y a una desproporción cefalopélvica. Estas pacientes se quedaron embarazadas a los 15 y 10 meses de la cirugía, respectivamente. Cinco pacientes fueron a FIV y dos de ellas lograron un embarazo de término, una por cesárea y otra por parto normal, a los 12 y 6 meses de la cirugía, respectivamente. En resumen, un $24 \%$ de las pacientes con deseo de embarazo y endometriosis intestinal lograron su objetivo por vía natural o mediante FIV.

En esta muestra se realizaron $23(44,2 \%)$ colonoscopías como parte del estudio preoperatorio; sin embargo, de 18 pacientes que presentaban rectorragia, solo en 10 se realizó una colonoscopía. Del total de las colonoscopías, 14 (61\%) informaron una lesión que protruía hacia el lumen intestinal con o sin estenosis. Respecto a las biopsias obtenidas en la colonoscopía, solo una informó la presencia de tejido endometrial en la lesión intestinal; el resto era normal o con inflamación inespecífica. No hubo lesiones neoplásicas. 
Tabla 2. Comparación entre los periodos 2001-2010 y 2011-2019

\begin{tabular}{|c|c|c|c|c|}
\hline & $\begin{array}{c}\text { Total } \\
(n=52)\end{array}$ & $\begin{array}{c}2001-2010 \\
(n=20)\end{array}$ & $\begin{array}{c}2011-2019 \\
(n=32)\end{array}$ & p \\
\hline Edad, años & $34,5 \pm 5,4$ & $33,6 \pm 5,8$ & $35 \pm 5,2$ & 0,36 \\
\hline Hemorragia digestiva baja & $18(34,6)$ & $9(45)$ & $9(28)$ & 0,21 \\
\hline Enema baritado & $14(28)$ & $13(65)$ & $1(3,3)$ & $<0,0001$ \\
\hline Tomografía computarizada & $11(21,6)$ & $9(45)$ & $2(6,5)$ & 0,001 \\
\hline Resonancia magnética & $25(50)$ & $4(20)$ & $21(70)$ & 0,001 \\
\hline Ecografía transvaginal & $32(62)$ & $0(0)$ & $32(100)$ & \\
\hline Laparoscopía & $39(75)$ & $7(35)$ & $32(100)$ & $<0,0001$ \\
\hline Resecciones segmentarias & $35(67,4)$ & $20(100)$ & $15(46,9)$ & $<0,0001$ \\
\hline Resecciones discoidales & $15(46,9)$ & $0(0)$ & $15(46.9)$ & \\
\hline Resección segmentaria y discoidal & $1(1,9)$ & $0(0)$ & $1(1,9)$ & \\
\hline Resección discoidal doble & $1(1,9)$ & $0(0)$ & $1(1,9)$ & \\
\hline Cirugías urgencia & 3 & 3 & 0 & \\
\hline Compromiso pared submucoso o mucoso & $20(38,5)$ & $15(75)$ & $5(15,6)$ & \\
\hline Reintervenciones & 1 & 1 & 0 & \\
\hline Días hospitalización & $4,8 \pm 0,3$ & $5,8 \pm 2,3$ & $4,1 \pm 0,9$ & 0,005 \\
\hline
\end{tabular}

\section{Tipo de cirugía realizada}

Se realizaron 52 cirugías con resección intestinal por endometriosis: 49 electivas y tres de urgencia. En los años 2001 a 2008, 13 casos fueron por vía laparotómica. En 2006 se comenzó a realizar las cirugías por vía laparoscópica, con dos conversiones a laparotomía ese año. A partir de 2009, todas las cirugía de endometriosis intestinal son por vía laparoscópica, sin otras conversiones $(n=39)$. Las lesiones intestinales endometriósicas se localizaban en el recto-sigmoides en 41 pacientes $(78,8 \%)$, en el sigmoides alto en $9(17,4 \%)$, y en el ciego y el íleon terminal en $2(3,8 \%$ ) (Tabla 3$)$.

Respecto a la técnica quirúrgica, $35(67,3 \%)$ de las 52 pacientes fueron sometidas a resección segmentaria, $15(28,8 \%)$ a resecciones discoidales simples, $1(1,9 \%)$ a resección discoidal doble y $1(1,9 \%)$ a ambas técnicas por lesión rectal baja (discoidal simple) y lesión sigmoidea alta (segmentaria) (Tabla 3). Desde 2011, debido a la mejora en su accesibilidad, todas las pacientes tienen rectoscopia intraoperatoria para evaluar las anatomosis, permitiendo la realización de pruebas neumáticas y la visualización y la hemostasia del sangrado de la anastomosis para así evitar reintervenciones.

Respecto al promedio de días de hospitalización, fue de $4,8 \pm 0,3$. Hasta el año 2010 , el promedio de días de cama por paciente fue de 5,8 $\pm 2,3$, y disminuyó a $4,1 \pm 0,9$ en promedio desde el año 2011, lo cual es estadísticamente significativo $(p<0,05)$.

Hasta el año 2010 hubo tres cirugías de urgencia por diagnóstico de obstrucción intestinal debida a endometriosis; posterior a este año, no se reportaron cirugías de urgencia. En estos tres casos se realizó una resección segmentaria con anastomosis sin ileostomía de protección. Una de ellas evolucionó con abdomen agudo secundario a una filtración de la anastomosis, siendo reoperada por laparoscopía al tercer día, realizándose reparación con sutura en la zona dehiscente, drenaje e ileostomía de protección. La paciente evolucionó favorablemente y se realizó reconstitución del tránsito intestinal a los 3 meses.

Respecto a las ileostomías, solo se realizaron dos. La primera fue la paciente que se acaba de describir (reintervención por filtración) y la segunda fue una ileostomía de protección electiva debido a una 
Tabla 3. Tipo de resección intestinal, ubicación e infiltración de la lesión endometriósica

\begin{tabular}{|l|c|}
\hline Tijpo cirugía $(\mathrm{n}=52)$ & $n(\%)$ \\
\hline Resección discoidal simple & $15(28,8)$ \\
\hline Resección discoidal doble & $1(1,9)$ \\
\hline Resección segmentaria & $35(67,3)$ \\
\hline Discoidal + segmentaria & $1(1,9)$ \\
\hline Segmento comprometido $(\mathrm{n}=52)$ & $n(\%)$ \\
\hline Rectosigmoides (bajo) & $41(78,8)$ \\
\hline Sigmoides (alto) & $9(17,4)$ \\
\hline Ileo cecal & $2(3,8)$ \\
\hline Anatomía patológica $(\mathrm{n}=52)$ & $n(\%)$ \\
\hline Mucosa & $4(7,7)$ \\
\hline Submucosa & $16(30,8)$ \\
\hline Muscular & $28(53,8)$ \\
\hline Subserosa & $2(3,8)$ \\
\hline Tejido graso perirrectal & $2(3,8)$ \\
\hline
\end{tabular}

resección segmentaria y anastomosis muy baja, a menos de $5 \mathrm{~cm}$ del margen anal. A esta paciente se le restituyó el tránsito al tercer mes de su cirugía, y en el cuarto mes presentó una estenosis de la anastomosis rectal que se dilató por rectoscopía de manera ambulatoria en policlínico, con posterior evolución favorable.

\section{Complicaciones quirúrgicas}

Se reportaron cuatro complicaciones posoperatorias, las cuales se describen detalladamente en la tabla 4. Hubo una reintervención dentro de los 30 días de la cirugía $(1,9 \%)$.

Las complicaciones se agruparon según la clasificación de Clavien-Dindo ${ }^{20}$, la cual se basada en el manejo posoperatorio de estas. Tres de ellas fueron de categoría II. La primera fue una paciente a quien se realizó una resección discoidal doble y presentó rectorragia, siendo tratada de manera satisfactoria con ácido tranexámico. La segunda paciente tuvo una resección discoidal simple asociada a una histerectomía, que posterior al alta reingresó con un síndrome febril y se le diagnosticó por TC de abdomen y pelvis una tromboflebitis parametrial, la cual se manejó con antibióticos intravenosos y tratamiento anticoagulante. Ambas pacientes evolucionaron satisfactoriamente.

La tercera complicación de categoría II fue en una paciente que, posterior a la resección segmentaria rectosigmoidea y tiflectomía, presentó rectorragia en el posoperatorio inmediato y requirió transfusión de dos unidades de glóbulos rojos, siendo observada por precaución en la unidad de intermedio quirúrgico por 24 horas. Luego se trasladó a sala y se mantuvo hospitalizada por 5 días, con una evolución satisfactoria.

Por último, tuvimos una complicación Clavien-Dindo IV A en una paciente operada por obstrucción intestinal de urgencia, que ya fue descrita anteriormente. A esta paciente se le realizó una rectosigmoidectomía con anastomosis término-terminal laparoscópica y fue reoperada a las 72 horas por un cuadro de abdomen agudo secundario a una filtración de la anastomosis, con posterior recuperación en la unidad de paciente crítico. Luego de 12 días de hospitalización, fue dada de alta con evolución satisfactoria. No se reportaron muertes en nuestra serie ni tampoco fístulas rectovaginales 0 urinarias.

\subsection{Resultados de anatomía patológica}

Los resultados obtenidos luego del análisis histopatológico confirmaron la endometriosis intestinal en el $100 \%$ de las muestras; no hubo lesiones neoplásicas malignas asociadas. Además, se evaluó el grado de infiltración de la lesión endometriósica en la pared intestinal, que se clasificó según la profundidad del compromiso de superficial a profundo (Tabla 3).

Se encontró que el 7,7\% tenían compromiso hasta la mucosa intestinal, el 30,8\% hasta la submucosa, el $53,8 \%$ hasta la capa muscular, el $3,8 \%$ solo subserosa y el $3,8 \%$ solo tejido graso perirrectal. Durante el periodo 2001-2010, todas las resecciones intestinales por endometriosis fueron segmentarias $(n=20)$, con un $75 \%$ de compromiso transmural (mucosa y submucosa). En cambio, a partir de 2011 hubo 32 resecciones intestinales (discoidales, doble discoidales, segmentarias), con un $15,6 \%$ de compromiso mucoso o submucoso. Esta diferencia del grado de infiltración es estadísticamente significativa $(p<0,05)$ (Tabla 2).

\section{Discusión}

La endometriosis intestinal tiene indicación quirúrgica cuando los síntomas de dolor pélvico (dismenorrea, dispareunia, disquecia) afectan negativamente la calidad de vida de la paciente. En nuestra serie se confirman 
Tabla 4. Complicaciones de las cirugías

\begin{tabular}{l|l}
\hline Año & Descripción \\
\hline Año 2010 & $\begin{array}{l}\text { La paciente ingresa para laparoscopía de urgencia por obstrucción intestinal. Se realiza una sigmoidectomía. En el } \\
\text { posoperatorio evoluciona con rectorragia e hidroneumoperitoneo. Se reinterviene por laparoscopía al tercer día debido } \\
\text { a una peritonitis difusa. Aseo, sutura, drenaje por laparoscopía e ileostomía. Dos días en cuidados intensivos, } 12 \text { días de } \\
\text { hospitalización. Reconstitución tránsito a los } 3 \text { meses. Clavien-Dindo IV A. }\end{array}$ \\
Año 2014 & $\begin{array}{l}\text { Cirugía laparoscópica programada, se realiza sigmoidectomía y tiflectomía con anastomosis rectoanal término-terminal } \\
\text { con stapler de } 29 \text { mm. Cursa con rectorragia importante en posoperatorio inmediato, se traslada a cuidado intermedio, } \\
\text { transfusión de dos unidades de glóbulos rojos, rectoscopía sin sangrado activo, evoluciona favorablemente. Cinco días } \\
\text { de hospitalización. Clavien-Dindo II. }\end{array}$ \\
Año 2016 & $\begin{array}{l}\text { Paciente operada de manera electiva, se le realiza doble resección discoidal con stapler de } 29 \text { mm y } 25 \text { mm por nódulo } \\
\text { de } 3 \text { a } 8 \text { cm del margen anal, con rectorragia que se trata con ácido tranexámico. Cinco días de hospitalización. }\end{array}$ \\
Clavien-Dindo II.
\end{tabular}

estos hallazgos ${ }^{4,6}$, observándose que el $90 \%$ de las pacientes presentaron dolor pélvico grave y que la mediana de este síntoma según la EVA era de 7 sobre 10 y la de dismenorrea de 9 sobre 10. Además, un tercio de ellas tenían infertilidad como diagnóstico asociado.

Actualmente, el diagnóstico de endometriosis intestinal se realiza mediante ecografía transvaginal extendida o RM pélvica, en las cuales es fundamental que el operador sea experto en endometriosis, tal como se describe en la literatura ${ }^{12,16,22-25}$. Creemos importante señalar el cambio en el estudio y el tratamiento de esta patología en nuestro centro a partir del año 2011, ya que entre 2001 y 2010 el diagnóstico se realizaba principalmente mediante TC de abdomen y pelvis, junto con un enema baritado, dado que las RM eran costosas y poco accesibles. Desde el año 2011, con la introducción de la unidad de endometriosis, se comienza a utilizar sistemáticamente la RM pelviana gracias a la disminución de su costo y la mayor accesibilidad. Además, las RM se evalúan y se discuten en conjunto con los radiólogos enfocados en esta patología. A partir del año 2015 se introduce la ecografía transvaginal extendida para endometriosis profunda, gracias a la formación específica de ginecólogos en esta área y los avances de la tecnología de ultrasonido ${ }^{25}$. Ambos cambios han permitido una mejora en la detección y el mapeo de la enfermedad, como se publicado en la literatura internacional ${ }^{4,25}$.

En las paciente con endometriosis que presentan rectorragia catamenial creemos importante agregar al estudio una colonoscopía, con el fin de hacer el diagnóstico diferencial con una neoplasia de origen intestinal ${ }^{4,6,26}$. De nuestras pacientes con rectorragia ( $n=18$ ), solo el $55,5 \%$ tenían el examen realizado al momento de la cirugía, porcentaje que creemos importante mejorar debido que es parte del estudio de la hemorragia digestiva baja. Actualmente, en el Hospital Clínico de la Universidad de Chile, y en relación con la mejor accesibilidad de este examen en el país, a todas las pacientes que tienen rectorragia se les solicita una colonoscopía, e incluso a aquellas que tienen un riesgo de resección intestinal para descartar otras patologías colónicas asociadas. Por otra parte, es interesante recalcar que solo una biopsia por colonoscopía mostró tejido endometrial; el resto fueron normales o con inflamación inespecífica. Esto concuerda con el 7\% de infiltración de la endometriosis en la mucosa intestinal que obtuvimos en las biopsias de las resecciones, tal como se ha observado también en otras series ${ }^{9,27}$. En este contexto, podemos afirmar que la colonoscopía nos ayudará a evaluar el grado de estenosis del lumen intestinal y a descartar una patología neoplásica intestinal cuando el cuadro clínico de dolor se presenta además con rectorragia catamenial, pero rara vez nos confirmará el diagnóstico de endometriosis rectosigmoidea, como ya se ha descrito en la literatura internacional ${ }^{4}$. Por otro lado, creemos importante señalar, basándonos en nuestra experiencia, que cuando las pacientes jóvenes (en general entre los 30 y 40 años) con dolor pelviano crónico son estudiadas mediante colonoscopía por otras causas, como el colon irritable, y el endoscopista digestivo detecta una protrusión fija en el lumen de la pared anterior rectosigmoidea, deben ser derivadas a ginecología (idealmente a una unidad 
de endometriosis) para su evaluación y descartar así una endometriosis profunda.

Previamente, en relación con la resección intestinal por endometriosis, la recomendación era un manejo radical de la enfermedad, similar al del cáncer colorrectal, con resección segmentaria amplia e ileostomía de protección. Esto generaba alteraciones funcionales propias de la cirugía radical y complicaciones en una mujer joven en búsqueda de fertilidad. En la actualidad, el tratamiento quirúrgico de la endometriosis intestinal busca ser más conservador, de acuerdo con las recomendaciones de las guías internacionales ${ }^{4,18}$. Hoy en día, toda paciente con una lesión intestinal baja (rectal y rectosigmoidea) tiene indicación de shaving o afeitado en un comienzo, independientemente del tamaño de la lesión, y si con este primer abordaje se considera que aún hay lesión residual, se recomienda realizar una resección discoidal, o en su defecto segmentaria si ninguna de las anteriores es técnicamente posible ${ }^{4,6,26}$. Por otra parte, si la lesión es sigmoidea, tiene indicación de resección segmentaria dado que el lumen intestinal es más estrecho, hay una mayor posibilidad de estenosis en el futuro y la tasa de complicaciones quirúrgica es más baja. Este cambio ha permitido disminuir los eventos adversos, como la filtración de la anastomosis y la fístula rectovaginal, desde un 3,5-25\% a menos de un 5\% en la actualidad $^{1,3,4,13-15,23}$. Esto se ve reflejado en nuestra muestra, en la que hasta el año 2010 todas las cirugías fueron resecciones segmentarias con hospitalizaciones significativamente más largas, con un promedio de hospitalización de 5,8 $\pm 2,3$ días. Posterior a ese año, con la inicio de la unidad de endometriosis, el diagnóstico mejoró y se introdujeron las técnicas conservadoras laparoscópicas, realizándose resecciones discoidales, doble discoidales y segmentarias cortas, con un promedio de hospitalización más corto, de 4,1 $\pm 0,9$ días $(p<0,05)$. Además, se puede observar que el $100 \%(n=20)$ de las cirugías realizadas entre los años 2001 y 2010 fueron resecciones intestinales segmentarias, con un $75 \%$ de compromiso transmural por invasión de la mucosa y la submucosa por el tejido endometrial. Esto contrasta con el resultado obtenido en las pacientes operadas posterior a ese año ( $n=32,2011-2019)$, en las que un $46,9 \%$ de las resecciones intestinales fueron segmentarias y solo un $15,6 \%$ tenía compromiso completo de la pared intestinal. Esta diferencia entre ambos periodos en cuanto a la infiltración transmural de las lesiones endometriósicas en la pared intestinal es estadísticamente significativa ( $p$ $<0,05$ ). De nuevo, esto refleja que en el periodo 20112019 las lesiones endometriósicas tienen menor infiltración transmural, hay menos operaciones por cuadros de obstrucción intestinal de urgencia, el tiempo de hospitalización es menor y la tasa de complicaciones es baja, probablemente en relación con un diagnóstico más precoz y el tratamiento con cirugía mínimamente invasiva.

En nuestra serie tuvimos cuatro complicaciones, de las cuales solo una (1,9\%) tuvo que ser reintervenida (Clavien-Dindo IV A) ${ }^{22}$, lo que concuerda con la literatura actual ${ }^{6,26}$. En este caso, la reintervención fue al tercer día por filtración de la anastomosis. La tasa de filtración de las anastomosis colorrectales por endometriosis está descrita entre un 3,5\% y un 25\% $\%^{6,13-15,28}$. Otra de las complicaciones graves reportadas es la fístula rectovaginal, cuya tasa es de un $2 \%$ aproximadamente ${ }^{4,6,26}$; sin embargo, en este estudio no hubo ningún caso. En nuestra serie, el porcentaje de complicaciones es bajo, probablemente debido a la detección más precoz de la enfermedad en esta última década, lo que permite un enfrentamiento electivo y planificado de estas pacientes, con un equipo ginecológico y colorrectal especializado en endometriosis, como se sugiere en la literatura internacional $1^{4,12,18}$.

Respecto a las ileostomías, en las pacientes con endometriosis intestinal, es importante destacar que hoy en día son poco frecuentes y fundamentalmente reservadas para resecciones muy bajas $(<5 \mathrm{~cm}$ del margen anal), anastomosis con tensión, mala vitalidad de los tejidos o reintervenciones por filtración $n^{4,18,28,29}$. En nuestra serie solo hubo dos casos $(3,8 \%)$, de los cuales uno fue cirugía electiva por resección segmentaria con anastomosis muy baja y el otro fue por una reintervención por filtración.

Es importante destacar, como resultado final, que de las pacientes con seguimiento la mayor parte no ha vuelto a presentar síntomas de dolor pelviano, excepto dos pacientes (3,8\%). Esto se puede deber a dos causas fundamentales: una, que la endometriosis es una enfermedad crónica con compromiso macroscópico y microscópico de la pelvis, y estos últimos focos pueden ser los que probablemente causen las recidivas o la mejoría incompleta de las pacientes ${ }^{9}$; y la otra razón importante de recidiva del dolor es por el proceso de sensibilización central del sistema nervioso y la disfunción miofascial del piso pelviano de las pacientes sometidas a dolor pélvico crónico, las cuales necesitan un tratamiento médico coadyuvante, psiquiatría, fisiatría, cinesioterapia del piso pelviano y neuroestimulación ${ }^{30,31}$. Es importante señalar las limitaciones del presente estudio retrospectivo en el seguimiento de los síntomas de dolor pelviano, ya que se basa en las evoluciones clínicas posoperatorias descritas por los médicos tratantes. 
A nuestro entender, esta es la serie más grande publicada en Chile de resecciones intestinales por endometriosis. Mordojovich et al..$^{18}$ describen su experiencia de 17 casos y Larraín et al..$^{19}$ la de sus 22 casos. Es importante recalcar que al inicio de esta cohorte, antes del año 2011, las lesiones endometriósicas intestinales tenían un compromiso de la pared más profundo, infiltrando hasta la mucosa, con lesiones más grandes y síntomas de obstrucción intestinal de urgencia, probablemente por la falta de diagnóstico y el desconocimiento de esta patología por parte de los médicos tratantes. Sin embargo, posterior a ese año, esto cambia gracias a la introducción de la unidad multidisciplinaria de endometriosis con RM pelviana y radiólogos expertos, ginecólogos y coloproctólogos especializados en esta área, y a partir del año 2015 con la ultrasonografía ginecológica extendida para endometriosis. Esto posibilitó una mejora en la detección y en el diagnóstico precoz de lesiones endometriósicas intestinales más pequeñas, cuya infiltración se sitúa en capas más superficiales de la pared del recto, disminuyendo así el porcentaje de pacientes con compromiso transmural. En consecuencia, permitió que hubiese menos operaciones por cuadros de obstrucción intestinal de urgencia, un tratamiento quirúrgico electivo con cirugías mínimamente invasivas cuyo resultado fue un menor tiempo de hospitalización y una baja tasa de complicaciones. Estos hallazgos demuestran que la introducción de la unidad multidisciplinaria de endometriosis permite un diagnóstico precoz y un tratamiento quirúrgico eficaz y oportuno, tal como se ha descrito en la literatura ${ }^{12,16}$. Nuestra unidad está compuesta por profesionales especializados en endometriosis (fisiatría, cinesiología, psicología, ginecología, coloproctología, urología), lo cual permite un adecuado tratamiento integral para pacientes con esta patología.

\section{Financiación}

Los autores declaran no haber recibido financiación para este estudio.

\section{Conflicto de intereses}

Los autores manifiestan no tener conflicto de intereses.

\section{Responsabilidades éticas}

Protección de personas y animales. Los autores declaran que para esta investigación no se han realizado experimentos en seres humanos ni en animales.
Confidencialidad de los datos. Los autores declaran que han seguido los protocolos de su centro de trabajo sobre la publicación de datos de pacientes.

Derecho a la privacidad y consentimiento informado. Los autores declaran que en este artículo no aparecen datos de pacientes.

\section{Bibliografía}

1. Donnez O, Roman $\mathrm{H}$. Choosing the right surgical technique for deep endometriosis: shaving, disc excision, or bowel resection? Fertil Steril. 2017;108:931-42

2. Roman H, Bubenheim M, Huet E, Bridoux V, Zacharopoulou C, Daraï E, et al. Conservative surgery versus colorectal resection in deep endometriosis infiltrating the rectum: a randomized trial. Hum Reprod. 2017;33:47-57.

3. Fuentes A, Escalona J, Céspedes P, Espinoza A, Johnson MC. Prevalence of endometriosis in 287 women undergoing surgical sterilization in Santiago Chile. Rev Med Chil. 2014;142:16-9.

4. Working Group of ESGE, ESHRE, and WES; Keckstein J, Becker CM, Canis M, Feki A, Grimbizis GF, Hummelshoj L, et al. Recommendations for the surgical treatment of endometriosis. Part 2: deep endometriosis. Hum Reprod Open. 2020;2020(1):hoaa002.

5. Kondo W, Bourdel N, Zomer MT, Slim K, Botchorischvili R, Rabischong B, et al. Surgery for deep infiltrating endometriosis: technique and rationale. Front Biosci (Elite Ed). 2013;5:316-32.

6. Abrao MS, Borrelli GM, Clarizia R, Kho RM, Ceccaroni M. Strategies for management of colorectal endometriosis. Semin Reprod Med. 2017;35:65-71.

7. Miranda-Mendoza I, Kovoor E, Nassif J, Ferreira H, Wattiez A. Laparoscopic surgery for severe ureteric endometriosis. Eur J Obstet Gynecol Reprod Biol. 2012;165:275-9.

8. Alves J, Puga M, Fernandes R, Pinton A, Miranda I, Kovoor E, et al. Laparoscopic management of ureteral endometriosis and hydronephrosis associated with endometriosis. J Minim Invasive Gynecol. 2017;24:466-72.

9. Kondo W, Bourdel N, Tamburro S, Cavoli D, Jardon K, Rabischong B, et al. Complications after surgery for deeply infiltrating pelvic endometriosis. BJOG. 2011;118:292-8.

10. Koninckx P, Ussia A. "Centers of excellence in endometriosis surgery" or "centers of excellence in endometriosis"? Gynecol Surg. 2010;7:109.

11. Abo C, Moatassim S, Marty N, Saint Ghislain M, Huet E, Bridoux V, et al. Postoperative complications after bowel endometriosis surgery by shaving, disc excision, or segmental resection: a three-arm comparative analysis of 364 consecutive cases. Fertil Steril. 2018;109:172-8.e1.

12. Tarjanne $S$, Heikinheimo $O$, Mentula M, Härkki $P$. Complications and long-term follow-up on colorectal resections in the treatment of deep infiltrating endometriosis extending to bowel wall. Acta Obstet Gynecol Scand. 2015;94:72-9.

13. Hernández Gutiérrez A, Spagnolo E, Zapardiel I, García-Abadillo Seivane R, López Carrasco A, Salas Bolívar P, et al. Post-operative complications and recurrence rate after treatment of bowel endometriosis: comparison of three techniques. Eur J Obstet Gynecol Reprod Biol X. 2019;4:100083.

14. Larraín D, Prado J, Buckel H, Mondión M, Veronesi V, Rojas I. Significant improvement in diagnosis and surgical management of deep-infiltrating endometriosis after formation of a specialized unit: a Chilean experience. J Gynecol Surg. 2018;35:3.

15. Ballester M, Roman H, Mathieu E, Touleimat S, Belghiti J, Daraï E. Prior colorectal surgery for endometriosis-associated infertility improves ICSI-IVF outcomes: results from two expert centres. Eur J Obstet Gynecol Reprod Biol. 2017;209:95-9.

16. Wattiez A, Puga M, Albornoz J, Faller E. Surgical strategy in endometriosis. Best Pract Res Clin Obstet Gynaecol. 2013;27:381-92.

17. Durruty G, Larraín D, Cuello M, Pomés C, Vidal H, Vergara A, et al. Endometriosis profunda del tabique rectovaginal con compromiso intestinal: manejo quirúrgico con resección segmentaria de rectosigmoides. Rev Chil Obstet Ginecol. 2008;73:192-203.

18. Mordojovich E, Espíndola L, Melkonian E, Espínola D, Villalón C Jensen $C$, et al. Surgical treatment of deep pelvic endometriosis with colorectal involvement. Revista de Cirugía. 2019;71:225-9.

19. Larraín D, Buckel H, Prado J, Abedrapo M, Rojas I. Multidisciplinary laparoscopic management of deep infiltrating endometriosis from 2010 to 2017: a retrospective cohort study. Medwave. 2019;19:e7750.

20. Dindo D, Demartines N, Clavien P. Classification of surgical complications. a new proposal with evaluation in a cohort of 6336 patients and results of a survey. Ann Surg. 2004;240:205-13.

21. Koninckx PR, Ussia A, Adamyan L, Wattiez A, Donnez J. Deep endometriosis: definition, diagnosis, and treatment. Fertil Steril. 2012;98:564-71. 
Rev Chil Obstet Ginecol. 2021;86(3)

22. Guerriero S, Condous G, van den Bosch T, Valentin L, Leone FPG, Van Schoubroeck D, et al. Systematic approach to sonographic evaluation of the pelvis in women with suspected endometriosis, including terms, definitions and measurements: a consensus opinion from the International Deep Endometriosis Analysis (IDEA) group. Ultrasound Obstet Gynecol. 2016;48:318-32.

23. Kondo W, Ribeiro R, Trippia C, Zomer MT. Laparoscopic treatment of deep infiltrating endometriosis affecting the rectosigmoid colon: nodulectomy or segmental resection? Gynecol Obstetric. 2013;S3:001.

24. Kavallaris A, Köhler C, Kühne-Heid R, Schneider A. Histopathological extent of rectal invasion by rectovaginal endometriosis. Hum Reprod. 2003;18:1323-7.

25. Badescu A, Roman H, Aziz M, Puscasiu L, Molnar C, Huet E, et al. Mapping of bowel occult microscopic endometriosis implants surrounding deep endometriosis nodules infiltrating the bowel. Fertil Steril. 2016;105:430-4.e26.

26. Clifford RE, Fowler H, Govindarajah N, Vimalachandran D, Sutton PA. Early anastomotic complications in colorectal surgery: a systematic review of techniques for endoscopic salvage. Surg Endosc. 2019;33:1049-65.
27. Khan AA, Wheeler JM, Cunningham C, George B, Kettlewell M, Mortensen NJ. The management and outcome of anastomotic leaks in colorectal surgery. Colorectal Dis. 2008;10:587-92.

28. Manríquez V, Sandoval C, Lecannelier J, Naser M, Guzmán R, Valdevenito $\mathrm{R}$, et al. Neuromodulación en patologías de piso pélvico. Rev Chil Obstet Ginecol. 2010;75:58-63.

29. Aredo JV, Heyrana KJ, Karp BI, Shah JP, Stratton P. Relating chronic pelvic pain and endometriosis to signs of sensitization and myofascial pain and dysfunction. Semin Reprod Med. 2017;35: 88-97.

30. Bassi MA Andres MP Bassi CM, Neto JS, Kho RM, Abrão MS Postoperative bowel symptoms improve over time after rectosigmoidectomy for endometriosis. J Minim Invasive Gynecol. 2020;27: 1316-23.

31. Burgos N, Miranda-Mendoza I, Gennero G, Alarcón C, Preisler J, Paredes $\mathrm{V}$, et al. Diagnóstico ecográfico de endometriosis profunda, experiencia Hospital Clínico Universidad de Chile. Rev Sochumb. 2019;22: 35-7. 